\title{
Metabolic Engineering of Microbial Cell Factories for Biosynthesis of Flavonoids: A Review
}

\author{
Hanghang Lou ${ }^{1,+}$, Lifei Hu ${ }^{2,+}$, Hongyun Lu ${ }^{1}$, Tianyu Wei ${ }^{1}$ and Qihe Chen ${ }^{1, *(D)}$ \\ 1 Department of Food Science and Nutrition, Zhejiang University, Hangzhou 310058, China; \\ louhanghang@zju.edu.cn (H.L.); luhongyun@zju.edu.cn (H.L.); 21913067@zju.edu.cn (T.W.) \\ 2 Hubei Key Lab of Quality and Safety of Traditional Chinese Medicine \& Health Food, \\ Huangshi 435100, China; lfyhu@hotmail.com \\ * Correspondence: chenqh@zju.edu.cn; Tel.: +86-0571-8698-4316 \\ + These authors are equally to this manuscript.
}

Citation: Lou, H.; Hu, L.; Lu, H.; Wei T.; Chen, Q. Metabolic Engineering of Microbial Cell Factories for Biosynthesis of Flavonoids: A Review. Molecules 2021, 26, 4522. https:// doi.org/10.3390/molecules26154522

Academic Editor: Luciana Mosca

Received: 5 July 2021

Accepted: 25 July 2021

Published: 27 July 2021

Publisher's Note: MDPI stays neutral with regard to jurisdictional claims in published maps and institutional affiliations.

Copyright: (c) 2021 by the authors. Licensee MDPI, Basel, Switzerland This article is an open access article distributed under the terms and conditions of the Creative Commons Attribution (CC BY) license (https:// creativecommons.org/licenses/by/ $4.0 /)$.

\begin{abstract}
Flavonoids belong to a class of plant secondary metabolites that have a polyphenol structure. Flavonoids show extensive biological activity, such as antioxidative, anti-inflammatory, anti-mutagenic, anti-cancer, and antibacterial properties, so they are widely used in the food, pharmaceutical, and nutraceutical industries. However, traditional sources of flavonoids are no longer sufficient to meet current demands. In recent years, with the clarification of the biosynthetic pathway of flavonoids and the development of synthetic biology, it has become possible to use synthetic metabolic engineering methods with microorganisms as hosts to produce flavonoids. This article mainly reviews the biosynthetic pathways of flavonoids and the development of microbial expression systems for the production of flavonoids in order to provide a useful reference for further research on synthetic metabolic engineering of flavonoids. Meanwhile, the application of co-culture systems in the biosynthesis of flavonoids is emphasized in this review.
\end{abstract}

Keywords: flavonoids; metabolic engineering; co-culture system; biosynthesis; microbial cell factories

\section{Introduction}

Flavonoids are a kind of secondary metabolite produced by plants in the process of long-term natural selection. They are widely found in vegetables, fruits, and medicinal plants. Structurally, they possess a C6-C3-C6 carbon framework, which is formed by connecting two aromatic rings (rings $\mathrm{A}$ and $\mathrm{B}$ ) through a heterocyclic ring that contains three carbon atoms (ring C) [1] (Figure 1). Thus far, about 10,000 flavonoids have been discovered, which comprise one of the largest families of natural products [2]. There are two forms of flavonoids in nature, one occurs in aglycone form in plants, and the other combines with sugar to form glycosides [3]. Common sugars attached to flavonoids include D-glucose, D-galactose, L-rhamnose, D-xylose, D-glucuronic acid, sophorose, rutinose, tangerine peel sugar, and gentianose [3,4]. Flavonoids normally occur in plants as O-glycosylated derivatives [5], and sugars are mostly attached to the hydroxyl groups at position 3 of the C-ring and positions 5 and 7 of the A-ring. However, some plants, such as maize, wheat, and rice, also produce C-glycosylated flavonoids [5]. These flavonoids play important roles in plants, animals, and humans. In plants, flavonoids are responsible for flower color and aroma and attract pollinators to disperse fruit and help plants propagate [6]. In addition, they also help plants combat various biotic and abiotic stresses, including ultraviolet protection in leaves, protection from microbial infections and herbivores, and defending from physical and radical damage $[7,8]$. As a kind of phytochemicals, flavonoids cannot be synthesized by humans or animals. Therefore humans generally absorb flavonoids from fruits, vegetables, legumes, and other foods, and flavonoids are thought to have health-promoting properties. Most studies have found that flavonoids have multiple physiological and pharmacological activities, such as antioxidative, anti-inflammatory, anti-mutagenic, anti-cancer, antiviral, 
antibacterial, and protecting the heart and cerebrovascular vessels $[6,9,10]$. Therefore, flavonoids show extensive applications in functional foods and some medicine. In fact, some flavonoid extracts, such as Masquelier's grape seed extract, have been sold as dietary supplements [11].<smiles>O=c1cc(-c2ccccc2)oc2ccccc12</smiles>

Figure 1. General structure of flavonoids.

The wide application of flavonoids has led to their increasing demand. At present, flavonoids are generally extracted from plants. However, plants have a long culture period, and their growth is also limited by the temperature and season. Additionally, the process of extraction and purification could increase the cost of production and lead to the loss of the product and the reduction of product activity [12]. Therefore, it is a huge challenge to produce these compounds on a large scale through plant extraction to meet medical and nutritional needs [13]. The total synthesis of some flavonoids has been realized by chemical approaches; however, this method required some extreme reaction conditions, such as high temperature, strong acid, strong base, and the use of heavy metal, which is unfriendly to the environment and hindered its scale-up and commercial application. In recent years, the production of flavonoids by microbial cell factories has attracted wide attention with the development of metabolic engineering tools, the in-depth studies on the synthesis pathway of flavonoids and available substrates $[7,12,14]$. Engineering the whole biosynthetic pathway to produce flavonoids in microorganisms has many advantages, such as a short production cycle, low waste production, low energy requirements, and mass production. Some microorganisms, such as Escherichia coli (E. coli), Saccharomyces cerevisiae (S. cerevisiae), Yarrowia lipolytica (Y. lipolytica), grow faster, and various genetic manipulations of this species and metabolic modifications have been studied in detail, so it has become possible to use these microorganisms as hosts for the production of flavonoids [2,15-17]. In a previous study, the production of flavonoids by genetically engineered bacteria had not been achieved, and one of the barriers to produce these compounds was the difficulty in the expression of active C4H. In 2003, a 4CL (4-coumarin-CoA ligase) was discovered in the gram-positive filamentous bacterium Streptomyces coelicolor [18]. The enzyme not only activated cinnamic acid to cinnamoyl-CoA but also activated 4-coumaric acid to 4-coumaroyl-CoA. The use of the enzyme would bypass the $\mathrm{C} 4 \mathrm{H}$ step for the production of pinocembrin chalcone from phenylalanine via the phenylpropanoid pathway [18]. In this study, the synthesis of flavonoids in microorganisms was achieved for the first time when PAL (phenylalanine ammonia lyase), CHS (chalcones synthase), and 4CL (4-coumarin-CoA ligase) were engineered in E. coli to produce naringenin and pinocembrin [18]. Since then, more and more enzymes related to flavonoid synthesis from different plants and microorganisms have been discovered and well-characterized [7], and meanwhile, various flavonoid pathways have been reconstituted in microbial species, including E. coli, S. cerevisiae, Streptomyces albus (S. albus), and Streptomyces coelicolor (S. coelicolor), to produce naringenin, eriodyctiol, pinocembrin, anthocyanins, scutellarein, baicalein, myricetin, kaempferol, quercetin, liquiritigenin, resokaempferol, fisetin, and so forth $[13,16,17,19,20]$. However, some problems, including the limitation of the availability of pathway genes, the instability of engineered strains, and the low yield of products in the synthesis process, still hinder the microbial production of flavonoids [1]. Several strategies have been employed to solve these problems, including the optimization of culture conditions, modular coculture technology, enhancement of malonyl-CoA, exploration of different substrates, optimization of functional expression of plant-derived P450 enzymes and fine-tuning the 
synthetic pathway using an iterative high-throughput screening method [21,22]. Among them, microbial co-cultures are gradually being investigated in-depth due to their many advantages over mono-cultures in terms of eliminating pathway bottlenecks and improving metabolic efficiency. In this review, the structural features, classification of flavonoids, and biosynthesis of flavonoids in plants are introduced in detail. Then, the biosynthesis of flavonoids in mono-cultures and co-cultures is discussed.

\section{Structural Features and Classification}

According to structural differences, such as the variation of the $\mathrm{C}$-ring and the position of the B-ring, flavonoids can be classified into flavones, flavonols, isoflavones, anthocyanins, chalcone, aurones, neoflavanoids, and their dihydrogen derivatives [6,7] (Table 1). For example, the structural feature of chalcones and dihydrochalcones is the absence of a C-ring in the basic flavonoid skeleton structure [7]. The C-ring of aurones is a five-membered carbon ring [7]. The C-ring of other flavonoids is mainly a six-membered carbon ring. Flavonoids in which a B-ring is connected to position 3 of a C-ring are called isoflavonoids, while those in which the B-ring is connected to position 4 are called neoflavonoids [7]. The B-ring of other flavonoids is mainly linked to position 2 of the C-ring [7]. Meanwhile, the types of flavonoids become diversified due to the chemical modifications by the differential placement of phenolic hydroxyl groups and other functional group modifications, such as methylation, methoxylation, glycosylation, acylation, malonylation, and so on [23].

Table 1. Structural features and classification of flavonoids.

\begin{tabular}{|c|c|c|}
\hline Groups & Structures & Examples \\
\hline Flavones & & $\begin{array}{l}\text { Apigenin, luteolin, baicalein, } \\
\text { and tangeritin }\end{array}$ \\
\hline Flavanones & & $\begin{array}{l}\text { Liquiritigenin, hesperitin, } \\
\text { naringenin, and eriodictyol }\end{array}$ \\
\hline Flavonols & & $\begin{array}{l}\text { Kaempferol, quercetin, } \\
\text { myricetin, and rutin }\end{array}$ \\
\hline Flavanonols & & $\begin{array}{l}\text { Aromadendrin, distylin, } \\
\text { dihydrokaempferol, and } \\
\text { dihydroquercetin }\end{array}$ \\
\hline
\end{tabular}


Table 1. Cont.

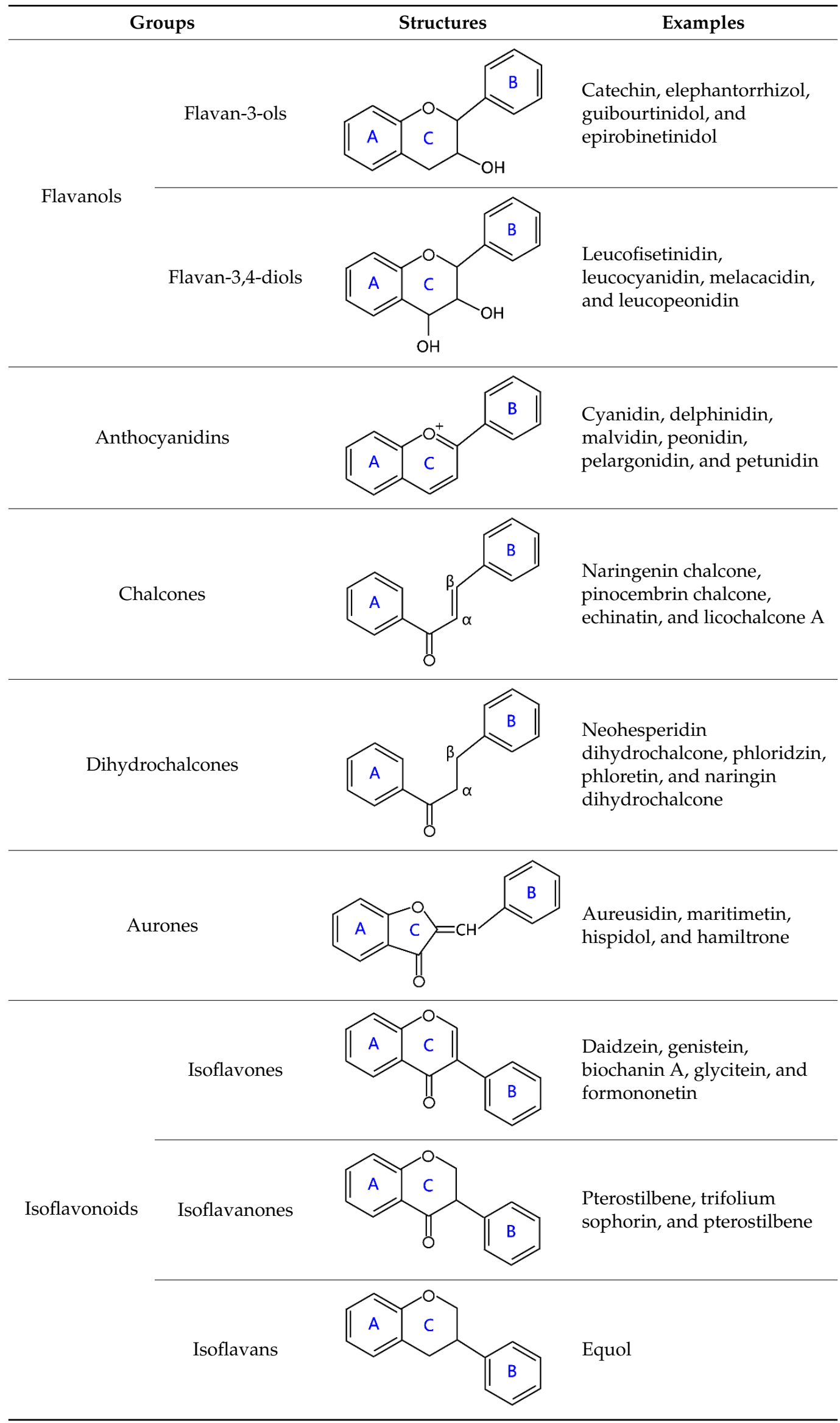


Table 1. Cont.

Groups Structures

\subsection{Flavones and Flavanones}

Structurally, flavones have a ketone in position 4 of the C-ring and a double bond between the second and third carbon atoms. The B-ring of flavones is linked in position 2 of the C-ring. These compounds are widely found in some plants encompassing celery, parsley, red peppers, chamomile, mint, and ginkgo biloba [6]. In particular, some natural flavones simultaneously have hydroxyl groups in positions 5 and 7 of the A-ring, and the $3^{\prime}$ and $4^{\prime}$ sites of the B-ring often have hydroxyl or methoxy groups. Their representative compounds are apigenin, luteolin, baicalein, and tangeritin (Table 1). Flavones also are present as glycosides, and rhamnose or glucose is generally attached to position 7 of the A-ring in most flavone glycosides.

Flavanones, also called dihydroflavones, are dihydrogen derivatives of flavones. They have the saturated C-ring, which has no conjugation between both rings $\mathrm{A}$ and B [6]. There is a chiral carbon atom in flavanones, which are optically active. They are widely distributed in some higher plant families, such as Compositae, Leguminosae, and Rutaceae [24]. Flavanones derived from the citrus fruits have been widely investigated due to interesting pharmacological effects, such as antioxidant, anti-inflammatory, blood lipid-lowering, and cholesterol-lowering agents, in which hesperitin, naringenin, and their derivatives are characteristic compounds [24] (Table 1). The main source of hesperitin and its derivative are sweet orange, tangelo, lemon, and lime, while naringenin and its derivative mainly come from grapefruit and sour orange [24,25]. Naringenin is a precursor of other classes of flavonoids, such as isoflavones, flavanols, anthocyanins, flavonols, and flavones [26,27]. The juice and peel of citrus fruits have bitterness because of the presence of these compounds [28].

\subsection{Flavonols and Flavanonols}

Flavonols are one of the important subgroups of flavonoids. They are the most abundant and widely distributed subgroup of flavonoids in comparison to other flavonoids [6]. Compared with flavones, flavonols have a hydroxyl group in position 3 of the C-ring, which may also be attached to O-glycosides [6]. Among the flavonols that have been discovered, there are more than ten kinds of sugars bound to flavonols, such as glucose, rhamnose, galactose, sophorose, and rutabiose, most of which are monosaccharides, but also disaccharides or trisaccharides. Some fruits, vegetables, and teas, such as onions, kale, lettuce, tomatoes, broccoli, apples, plums, grapes, and berries, are the major sources of flavonols. The most studied flavonols are kaempferol, quercetin, myricetin, and rutin (Table 1).

Flavanonols, also called dihydroflavonol, are dihydrogen derivatives of flavonols, which are the 3-hydroxy derivatives of flavanones. They have a positive effect on human health as they prevent the accumulation of free radicals and have anti-inflammatory, antioxidant, and antidiabetic activities [29]. Dihydroflavonols exist widely in some plants, such as Pseudotsuga menziesii, and Larix gmenlinii $[30,31]$. Aromadendrin, distylin, dihydrokaempferol, and dihydroquercetin are examples of this class of flavonoids (Table 1). 


\subsection{Flavanols}

Flavanols are a complex subclass of flavonoids, which include a variety of monomeric, oligomeric and polymeric compounds. The monomeric forms of flavanols are catechins, mainly encompassing catechin (C), epicatechin (EC), epigallocatechin (EGC), epicatechin gallate (ECG), and epigallocatechin gallate (EGCG), while the oligomeric or polymeric compounds are proanthocyanidins [32]. Among them, the polymers composed exclusively of catechin or epicatechin are called procyanidins [33]. Flavanols are abundant in cocoa, green tea, grapes, apples, and red wine, and cocoa flavanols are extensively studied owing to their higher flavanol content than other flavonoid-containing products, such as tea and wine $[34,35]$. These compounds have been observed to exert beneficial effects on reducing the risk of suffering from chronic diseases (cardiovascular diseases, metabolic disorders, cancer) [36]. From a structural point of view, flavanols lack a ketone oxygen in position 4 of the C-ring and the double bond between positions 2 and 3 of the $C$-ring, but they have multiple hydroxyl groups on A, B, and C-rings. In particular, flavanols in which there is a hydroxyl group in position 3 of the C-ring are called flavan-3-ols (also known as catechins), while flavanols are also referred to as flavan-3,4-diols, as position 3,4 of the C-ring has a hydroxyl group, respectively [12]. Catechin, elephantorrhizol, guibourtinidol, and epirobinetinidol belong to flavan-3-ols, and leucofisetinidin, leucocyanidin, melacacidin, and leucopeonidin belong to flavan-3,4-diols (Table 1).

\subsection{Anthocyanidins}

Anthocyanidins belonging to the flavonoid family are one of the most common watersoluble pigments in nature. In plants, anthocyanidins are always glycosylated and exist in the form of anthocyanins, and they are responsible for the vibrant color (red, orange, blue, purple) of fruits, flowers, leaves, vegetables, and grains [37]. The color of these compounds depends not only on $\mathrm{pH}$, but is also affected by methylation or acylation at the hydroxyl groups on the A and B-rings [38]. Usually, they are used to color food for substitution of synthetic colorants in the food industry. Meanwhile, anthocyanidins are beneficial to human health because of their antioxidant, anti-obesity, anti-inflammatory, and anti-carcinogenic properties [37]. Anthocyanidins and anthocyanins are unstable and easily affected by many factors, such as temperature, oxygen, $\mathrm{pH}$, light, metal ions, and enzymes [39]. Structurally, anthocyanidins have the basic structure of flavylium ion but are not equipped with a ketone oxygen in position 4 of the C-ring [37]. Cyanidin, delphinidin, malvidin, peonidin, pelargonidin, and petunidin are the most common anthocyanidins distributed in plants (Table 1).

\subsection{Chalcone}

Unlike many flavonoids, chalcones are open-chain flavonoids with two aromatic rings linked by a three-carbon enone moiety [40]. Chemically, chalcones possess a 1,3-diphenyl2-propenon core structure, which is divided into hydroxychalcones, methoxychalcones, aminochalcones, arylchalcones, alkylchalcones, nitrogenous chalcones, and others according to the different substituents $[40,41]$. Chalcones are biogenetic precursors of flavones. Chalcone is an important plant pigment, and it can provide a yellow color to the petals of some medicinal plants due to a very conjugated system in its skeleton [42]. Chalcones are abundantly present in strawberries, bearberries, tomatoes, pears, and certain wheat products [6]. Naringenin chalcone, pinocembrin chalcone, echinatin, and licochalcone A are prominent members of the chalcone family (Table 1). Chalcones have attracted wide attention because of their numerous nutritional and pharmacological activities, such as antibacterial, antinociceptive, anticonvulsant, and anti-inflammatory. The dihydro derivatives of chalcones are dihydrochalcones, some examples of which are neohesperidin dihydrochalcone, phloridzin, phloretin, and naringin dihydrochalcone (Table 1). Neohesperidin dihydrochalcone (NHDC), widely extracted from natural citrus plants, is an important dihydrochalcone, and it has been widely studied as a food additive because of its sweet taste [43]. 


\subsection{Aurones}

Aurones represent a minor class of flavonoids, which was discovered in 1943 and named "aurone" (from Latin aurum, "gold") by Bate-Smith and Geissman in 1951 [44]. Structurally, aurones contain a benzofuranone core and a phenyl group linked by a carboncarbon exocyclic double bond [44]. They are regarded as isomers of flavones and isoflavones and also can be synthesized by the oxidative cyclization of 2'-hydroxychalcones [45]. Aurones occur in flowers of Asteraceae, Plantaginaceae, Oxalidaceae, Gesneriaceae, Rosaceae and others, tree heartwoods of Fabaceae, Anacardiaceae, and Rhamnaceae, seeds and roots of non-tree Fabaceae, Moraceae, and other families [44]. Currently, more than one hundred compounds have been isolated from plants and fully investigated, including their glycosylated derivatives, auronols, and dimers. It is worth noting that aurones not only have advantages for plants, such as flower coloration and pollination, photoprotection, and antiinfection, but also show a wide range of biological activity, including antiviral, antibacterial, antifungal, anti-inflammatory, antitumor, antimalarial, antioxidant, neuropharmacological activities, and so on [46].

\subsection{Isoflavonoids}

Isoflavonoids are a distinctive subgroup of flavonoids existing in abundance in plants belonging to the families Fabaceae and Iridaceae [47]. Some isoflavonoids have also been reported to be isolated from fungi and actinomycetes [48,49]. Isoflavonoids possess a molecular framework formed by 15 carbon atoms with the general structure of $\mathrm{C} 6-\mathrm{C} 3-\mathrm{C} 6$, but unlike other flavonoids (such as flavones, flavonols, flavanones), the B-ring of isoflavonoids is attached to position 3 of the C-ring. Isoflavonoids are divided into isoflavones, isoflavanones, isoflavans and so on [7]. Because their structure is similar to estrogen, they can interact with estrogen receptors and exhibit weak estrogenic activity, so isoflavonoids are sometimes called phytoestrogens [50]. In addition, isoflavonoids are extensively reported for their other biological and pharmacological activities, including antidiabetic, antibacterial, antifungal, antiviral, antitumor, anti-inflammatory, and antiaging [48]. Examples of most studied isoflavonoids that have been displayed include daidzein, genistein, biochanin A, glycitein, and formononetin (Table 1).

\subsection{Neoflavonoids}

Neoflavonoids belong to a further small subclass of flavonoids, and they also have the general structure of $\mathrm{C} 6-\mathrm{C} 3-\mathrm{C} 6$ with the B-ring connected to position 4 of the C-ring. Neoflavonoids contain neoflavone (4-arylcoumarin), 3,4-dihydro-4-arylcoumarins, and neoflavene (4-arylchromene) [7]. Neoflavonoids are present in a wide variety of plants from families Passifloraceae, Asteraceae, Clusiaceae, Leguminosae, Rubiaceae, and Rutaceae [51]. Calophyllolide isolated in 1951 from extracts of Calophyllum inophyllum seeds was the first new neoflavonoid from natural sources [52]. Dalbergin is the most common and widely distributed neoflavone in plants [52].

\subsection{Biflavonoids}

Structurally, biflavonoids are characterized as dimeric flavonoids, which generally comprise dimers of flavone-flavone, flavone-flavonone, flavonone-flavonone, chalconeisoflavone, and flavanone-chalcone [53]. The individual flavones in the dimer are directly interconnected by $\mathrm{C}-\mathrm{C}$ or C-O-C bonds [53]. Amentoflavone and robustaflavone belong to biflavonoids connected by the $\mathrm{C}-\mathrm{C}$ bond, while ochnaflavone and hinokiflavone are examples of biflavonoids connected via the $\mathrm{C}-\mathrm{O}-\mathrm{C}$ bond [54]. Biflavonoids are found in plants such as Selaginella tamariscina, Ginkgo biloba, Cephalotaxus koreana, Nandina domestica, and Lonicera japonica [54]. The first biflavone isolated from natural sources was ginkgentin. It was isolated in 1929 from Ginkgo biloba L. as a yellow pigment [55]. Biflavonoids exhibit extensive pharmacological properties, including anti-inflammatory, antioxidant, inhibitory activity against phospholipase A2 (PLA2), and antiprotozoal activity [55]. 


\section{Biosynthesis of Flavonoids in Plants}

As a kind of secondary plant metabolite, the biosynthesis pathway of flavonoids in plants has been well studied. Understanding the biosynthesis of flavonoids is essential to enrich the resources of flavonoids and increase the production of flavonoids. Flavonoids are synthesized through the phenylpropanoid pathway [56]. The initial precursors of the phenylpropanoid pathway are L-phenylalanine or L-tyrosine, which are generally produced through the shikimate and aerogenate pathways [7]. The primary metabolite glucose produces phosphoenolpyruvate (PEP) and erythrose 4-phosphate (E4P) through the glycolytic pathway and pentose phosphate pathway, respectively, and they react to produce shikimic acid [57]. After a series of enzymatic reactions, shikimic acid can produce chorismate, prephenate, and finally L-phenylalanine and L-tyrosine [7] (Figure 2).

The phenylpropanoid pathway is a ubiquitous and well-described pathway for plant secondary metabolites. The first three common steps of the phenylpropanoid pathway are catalyzed by phenylalanine ammonia lyase (PAL), cinnamic acid 4-hydroxylase $(\mathrm{C} 4 \mathrm{H})$, and 4-coumarolyl-CoA ligase (4CL) [1]. Phenylalanine ammonia lyase (PAL) is responsible for converting L-phenylalanine into cinnamic acid, which is then oxidized by cinnamic acid 4-hydroxylase $(\mathrm{C} 4 \mathrm{H})$ to $p$-coumaric acid [56]. 4-coumarin-CoA ligase (4CL) converts $p$-coumaric acid into $p$-coumaroyl-CoA, which is one of the precursors of $\mathrm{C} 6-\mathrm{C} 3-\mathrm{C} 6$ backbone biosynthesis of different flavonoid classes [12]. However, the pathway of L-tyrosine conversion is shorter than the pathway using L-phenylalanine as the starting precursor. L-tyrosine can be directly catalyzed by tyrosine ammonia lyase (TAL) to $p$-coumaric acid, bypassing the $\mathrm{C} 4 \mathrm{H}$ intermediate [12]. Then, a molecule of $p$-coumaroyl CoA and three molecules of malonyl-CoA are condensed to form naringenin chalcone catalyzed by chalcones synthase (CHS), which is an initial reaction in the synthesis of flavonoid classes in the phenylpropanoid pathway [7]. Malonyl-CoA is mainly produced by acetyl-CoA via the catalysis of acetyl-CoA carboxylase (ACC) [7]. Subsequently, the chalcone backbone is catalyzed by different classes of enzymes (such as isomerase, hydroxylase, oxido-reductase, transferase) to produce other flavonoid subgroups [8]. Chalcones can be converted into flavanones by chalcone isomerase (CHI) or to aurones by plant polyphenol oxidase (PPO) [8]. However, flavanones can not only be hydroxylated by flavanone $3-\mathrm{OH}$ transferase $(\mathrm{F} 3 \mathrm{H})$ to produce flavanonols but can also be converted into flavones with a double bond between the C-2 and C-3 (of the C-ring) through the enzymatic action of flavone synthase (FNS) [58]. In addition, flavanones can also be converted into isoflavones via isoflavone synthase (IFS) [58]. Next, flavonol synthase (FLS) can oxidize flavanonols to flavonols, while dihydroflavonol-4-reductase (DFR) can reduce flavanonols to give rise to flavan-3,4-diols (also named leucoanthocyanidins) [58]. Finally, flavan-3,4-diols can be transformed into anthocyanidins by anthocyanidin synthase (ANS), and leucoanthocyanidin reductase (LAR) directly reduces flavan-3,4-diols to the corresponding flavan-3-ols [8]. These compounds are further modified into various flavonoids by glycosyltransferase, methyltransferase, acyltransferase, and so on [7]. 


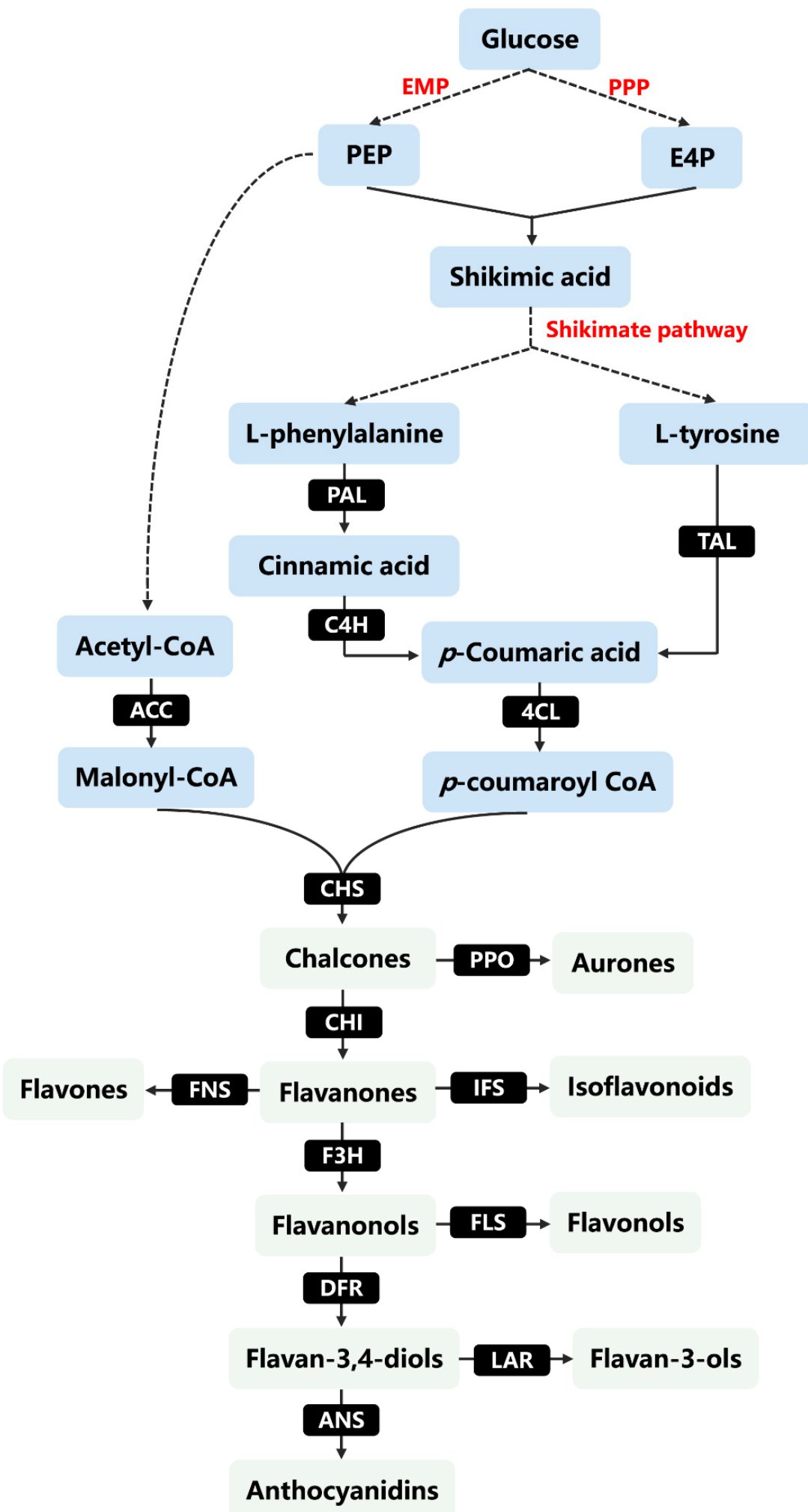

Figure 2. Biosynthesis pathway network of flavonoids in plants. EPM: glycolytic pathway; PPP: pentose phosphate pathway; PEP: phosphoenolpyruvate; E4P: erythrose 4-phosphate; PAL: phenylalanine ammonia lyase; $\mathrm{C} 4 \mathrm{H}$ : cinnamate 4-hydroxylase; TAL: tyrosine ammonia-lyase; 4CL: 4-coumarinCoA ligase; ACC: acetyl-CoA carboxylase; CHS: chalcone synthase; $\mathrm{CHI}$ : chalcone isomerase; PPO: polyphenol oxidase; F3H: flavanone 3-hydroxylase; IFS: isoflavone synthase; FNS: flavone synthase; DFR: dihydroflavonol 4-reductase; FLS: flavonol synthase; LAR: leucoanthocyanidin reductase; ANS: anthocyanidin synthase. 


\section{Biosynthesis of Flavonoids by Metabolic Engineering \\ 4.1. Microbial Mono-Culture for Flavonoid Synthesis}

Recently, microbial fermentation methods for the high-yield production of natural plant products have been extensively investigated. Table 2 shows the applications of microbial mono-culture for the production of flavonoids in the past five years. As shown in Table 2, E. coli and S. cerevisiae are commonly selected microbial hosts. E. coli is usually the host of choice because it has several advantages, including a high growth rate, established high cell density culture technology, and extensive investigations in genetic engineering [59]. As a GRAS (generally regarded as safe) strain, S. cerevisiae is often used as a product host, and it has a complete intracellular membrane system and is superior to E. coli in terms of the expression of membrane proteins [57]. In the process of de novo synthesis of anthocyanins by $S$. cerevisiae, a higher titer of eriodictyol was obtained, which was $152 \mathrm{mg} / \mathrm{L}$ [60]. Eriodictyol was formed by the hydroxylation of naringenin by the cytochrome P450 (CYP) enzymes flavonoid-3-hydroxylase $\left(\mathrm{F}^{\prime} \mathrm{H}\right)$. The high expression of $\mathrm{F}^{\prime} \mathrm{H}$ demonstrated the superior ability of $S$. cerevisiae to functionally express plant CYPs. In this study, pelargonidin-3-O-glucoside (P3G), cyanidin-3-O-glucoside (C3G), and delphinidin-3-O-glucoside (D3G) were synthesized from glucose in S. cerevisiae for the first time, and their titers were $0.85,1.55$, and $1.86 \mathrm{mg} / \mathrm{L}$, respectively [60]. When de novo biosynthesis of flavonoids is conducted by microorganisms, the efficient expression and targeting of pathway enzymes is the key to improving product yield. Apart from selecting suitable microbial hosts for heterologous expression of pathway enzymes, other strategies had been adopted. For example, when icariin was generated in S. cerevisiae, the pathway enzyme methyltransferase GmOMT2 would lose its activity due to the low $\mathrm{pH}$ in the cytoplasm if it was expressed in the cytoplasm [57]. In order to solve this problem, GmOMT2 could be relocated into the mitochondria (higher $\mathrm{pH}$ than cytoplasm) of S. cerevisiae. The synthesis of icaritin from glucose in microorganisms was also achieved for the first time, and the yield was $7.2 \mathrm{mg} / \mathrm{L}$ [57]. However, this regulation mechanism is still under elucidation for GmOMT2. The balance of biosynthetic pathways is another key to production optimization. In order to balance the metabolic flux, an iterative high-throughput balancing (IHTB) strategy was built to thoroughly fine-tune the naringenin biosynthetic pathway [22] It constructed all possible gene-promoter combinations and then screened them many times. After several rounds of high-throughput screening, the metabolic flux of naringin synthesis pathway reached equilibrium, and the final titer was $191.9 \mathrm{mg} / \mathrm{L} \mathrm{[22].}$

Malonyl-CoA is an important precursor for the production of flavonoids, and three molecules of malonyl-CoA and one molecule of $p$-coumaroyl-CoA were mixed to synthesize naringenin chalcone by chalcone synthase. At the same time, it is also an essential intermediate for the synthesis of fatty acids that support cell growth in microorganisms [58]. In order to efficiently produce flavonoids, it is important to balance the biosynthesis of flavonoids and cell growth. Recently, an induction-free system to de novo biosynthesize naringenin from glucose by E. coli was established, and this system could automatically balance the production of naringenin, the accumulation of $p$-coumaric acid, and cell growth [61]. Because the direct precursor of malonyl-CoA is acetyl-CoA, the biosynthesis of acetyl-CoA is closely related to carbon source and acetic acid, so carbon source and acetic acid can be used to regulate the synthesis of acetyl-CoA and then the synthesis of malonyl-CoA. In order to increase the production of malonyl-CoA at the fermentation level, the concentration of glucose, glycerol, and potassium acetate (KAc) in the medium was optimized [61]. After optimization, the yield of naringenin could reach $588 \mathrm{mg} / \mathrm{L}$, which was the highest titer reported in E. coli so far. In another study, a growth-coupled NCOMB (naringenin-coumaric acid-malonyl-CoA-balanced) DRN (dynamic regulation network) was developed, which could directly sense the accumulated naringenin and $p$-coumadin acid to dynamically regulate gene expression and finally achieve the goal of synchronizing cell growth and supplementing malonyl-CoA to form naringenin [62]. The yield of naringenin obtained by this method was $523.7 \mathrm{mg} / \mathrm{L}$. During the biosynthesis of flavonoids, glucose, glycerol, and some amino acids (L-tyrosine and L-phenylalanine) are generally used as the substrates to 
synthesize flavonoids in E. coli or S. cerevisiae. Other microorganisms (S. albus, Y. lipolytica, Corynebacterium glutamicum (C. glutamicum), Pichia pastoris (P. pastoris), and Lactococcus lactis (L. lactis)) have also been studied as microbial hosts, and some intermediate products (naringenin, taxifolin, afzelechin, and catechin) in the biosynthetic pathways of flavonoids and natural products (tea) have also been used as substrates [2,20,63-65]. In recent reports, anthocyanins were generated from green tea by engineered L. lactis strains, and the total titer of anthocyanins was $1.5 \mathrm{mg} / \mathrm{L}$ [65]. Specifically, the engineered strain not only produced the expected red-purple compounds cyanidin and delphinidin, but also orange and yellow pyranoanthocyanidins with unexpected methylation patterns after fermentation.

Table 2. Microbial mono-culture for the production of flavonoids in the past five years.

\begin{tabular}{|c|c|c|c|c|}
\hline Product & Substrate & Host Strain & Titer (mg/L) & Reference \\
\hline Luteolin & Glucose & S. albus & 0.09 & [66] \\
\hline Apigenin & Glucose & S. albus & 0.089 & [66] \\
\hline Apigenin & Glucose and naringenin & S. albus & 0.384 & [66] \\
\hline Scutellarin & Glucose & S. cerevisiae & 108 & {$[67]$} \\
\hline Apigenin-7-O-glucuronide & Glucose & S. cerevisiae & 185 & {$[67]$} \\
\hline Baicalein & L-phenylalanine & E. coli & 23.6 & [13] \\
\hline Scutellarein & L-tyrosine & E. coli & 106.5 & [13] \\
\hline Isoorientin & Luteolin & E. coli & 3829 & [68] \\
\hline Isovitexin & Apigenin & E. coli & 3772 & [68] \\
\hline Liquiritigenin & Glucose & S. cerevisiae & 5.31 & [16] \\
\hline Eriodictyol & Glucose & S. albus & 0.002 & [66] \\
\hline Eriodictyol & Naringenin & E. coli & 62.7 & [69] \\
\hline Eriodictyol & Caffeic acid & C. glutamicum & 37 & [63] \\
\hline Eriodictyol & Glucose & S. cerevisiae & 152 & {$[60]$} \\
\hline Eriodictyol & Glucose & Y. lipolytica & 54.2 & {$[70]$} \\
\hline Eriodictyol & Glucose & Y. lipolytica & 134.2 & [2] \\
\hline Eriodictyol & Glycerol & E. coli & 88 & [19] \\
\hline Homoeriodictyol & Glycerol & E. coli & 17 & [19] \\
\hline Pinocembrin & Glycerol & E. coli & 214 & [19] \\
\hline Naringenin & $p$-Coumaric acid & C. glutamicum & 35 & [63] \\
\hline Naringenin & Tyrosine & S. cerevisiae & 90 & {$[71]$} \\
\hline Naringenin & Tyrosine & E. coli & 191.9 & [22] \\
\hline Naringenin & Glucose & Y. lipolytica & 71.2 & [70] \\
\hline Naringenin & Glucose & $Y$. lipolytica & 252.4 & [2] \\
\hline Naringenin & $p$-Coumaric acid & S. cerevisiae & 648.63 & {$[72]$} \\
\hline Naringenin & Glycerol & E. coli & 484 & [19] \\
\hline Naringenin & Glucose & E. coli & 588 & [61] \\
\hline Naringenin & Glucose & E. coli & 523.7 & [62] \\
\hline Fisetin & Glucose & S. cerevisiae & 2.3 & [16] \\
\hline Resokaempferol & Glucose & S. cerevisiae & 14.54 & [16] \\
\hline Kaempferol & Glucose & S. cerevisiae & 26.57 & {$[16]$} \\
\hline Quercetin & Glucose & S. cerevisiae & 20.38 & [16] \\
\hline Quercetin & Glucose & S. albus & 0.599 & [20] \\
\hline Taxifolin & Glucose & Y. lipolytica & 48.1 & [70] \\
\hline Taxifolin & Glucose & Y. lipolytica & 110.5 & {$[2]$} \\
\hline Silybin and isosilybin & Eugenol and taxifolin & E. coli & 2580 & {$[73]$} \\
\hline Taxifolin & Glucose & S. cerevisiae & 336.8 & {$[74]$} \\
\hline Catechin & Afzelechin & E. coli & 34.7 & {$[69]$} \\
\hline Peonidin 3-O-glucoside & Catechin & E. coli & 56.3 & {$[75]$} \\
\hline Pelargonidin 3-O-glucoside & Glucose & S. cerevisiae & 0.85 & [60] \\
\hline Cyanidin 3-O-glucoside & Glucose & S. cerevisiae & 1.55 & [60] \\
\hline Delphinidin 3-O-glucoside & Glucose & S. cerevisiae & 1.86 & {$[60]$} \\
\hline Cyanidin 3-O-glucoside & Catechin & C. glutamicum & 40 & {$[76]$} \\
\hline Cyanidin 3-O-glucoside & Catechin & E. coli & 439 & {$[77]$} \\
\hline Anthocyanins & Tea & L. lactis & 1.5 & [65] \\
\hline Orobol & Genistein & P. pastoris & 23 & [64] \\
\hline $4^{\prime}$-O-methyl-genistein & Genistein & E. coli & 48.61 & {$[78]$} \\
\hline $4^{\prime}$-O-methyl-daidzein & Daidzein & E. coli & 102.88 & [78] \\
\hline Icaritin & Glucose & S. cerevisiae & 7.2 & [57] \\
\hline
\end{tabular}




\subsection{Microbial Co-Culture for Flavonoid Synthesis}

Since long ago, humankind has used natural communities to ferment foods and beverages, produce drugs and chemicals, and treat wastes [79]. However, only recently have scientists begun to investigate the application of co-culture techniques in metabolic engineering and synthetic biology. Modular co-culture engineering is to cultivate two or more microbial strains to complete a target biosynthetic pathway. The complete biosynthetic pathway is firstly modularized, and then each microorganism is responsible for a specific module. The type of relationship between the cultured microbial hosts can be commensalism, cooperation, amensalism, predation, and no interaction [80]. Compared with the traditional mono-culture method, microbial co-culturing greatly reduces the biosynthetic labor of each strain, lowers the related metabolic burden, and withstands greater environmental disturbances. Currently, microbial co-culture techniques have been applied for the production of various compounds, including fuels, amino acids, proteins, polyphenols (including flavonoids), alkaloids, terpenoids, and other chemicals [80,81].

Due to the clarification of the biosynthetic pathway of flavonoids, characterization of pathway enzymes, and wide application of flavonoids, flavonoids are more attentioned natural products through modular co-culture engineering biosynthesis, including genistein, naringenin, apigetrin, pelargonidin 3-O-glucoside, icaritin, and (+)-afzelecin, compared with other natural products (such as alkaloids and terpenoids) [81]. As shown in Table 3, cocultured strains are mostly different strains of the same organism, which may be due to the fact that the use of different strains within the same organism in multi-culture can simplify growth factors, antibiotics, and downstream processing. Microbial co-culture technology was employed for the production of flavonoids starting in 2007 [82]. The co-culture of $S$. cerevisiae carrying the IFS gene and E. coli with a high ability to produce (S)-naringenin (the direct precursor of genistein) from tyrosine could produce genistein. The yield of genistein was $6 \mathrm{mg} / \mathrm{L}$, which was 17.6 times higher than that of genistein produced by mono-culture of $S$. cerevisiae $(0.34 \mathrm{mg} / \mathrm{L})$ [82]. This is mainly due to the reduction of metabolic stress and the use of an appropriate host to increase pathway enzyme activity. After optimization of co-culture conditions, the titer of genistein was increased to $100 \mathrm{mg} / \mathrm{L}$ [83]. Naringenin is the central precursor of most flavonoids, and its synthesis by co-culture using different substrates has been reported. When naringenin was synthesized de novo from glucose through the E. coli-E. coli co-culture system, the biosynthesis pathway of naringenin was split into two modules [84]. An E. coli strain (known as the upstream strain) was designed to convert glucose into tyrosine or $p$-coumaric acid (module 1), and the other E. coli strain (known as the downstream strain) was constructed to be responsible for the conversion of tyrosine or $p$-coumaric acid to naringenin (module 2). The relationship between the two strains was commensalism. Meanwhile, some strategies, including screening E. coli strains and optimizing IPTG induction time and the inoculation ratio of co-culture strains, were adopted to adjust and balance the biosynthetic intensity between different pathway segments. Finally, the yield of naringenin was increased to $41.50 \mathrm{mg} / \mathrm{L}$. In addition, naringenin has been synthesized by the co-culture of E. coli and S. cerevisiae, and coculture of $S$. cerevisiae and $S$. cerevisiae using xylose and $p$-coumaric acid as substrates, respectively $[85,86]$. However, the titers were lower than that of naringenin produced by the co-culture of E. coli and E. coli. Notably, the co-culture of S. cerevisiae and S. cerevisiae strains separately containing AlCHS and Al4CL could produce not only naringenin but also resveratrol [86]. An artificial icaritin biosynthetic pathway was initially designed to generate icaritin in microbes this year [57]. During the biosynthesis of icariin, the role of methyltransferase GmOMT2 was very important. GmOMT2 was sensitive to low $\mathrm{pH}$ and loses its activity when expressed in yeast cytoplasm; this was the widespread issue of incompatible $\mathrm{pH}$ conditions encountered in basic and applied bioproduction research. In this study, an E. coli strain expressing GmOMT2 and the 8-prenylkaempferol-producing $S$. cerevisiae strain were co-cultured to overcome the difficulty. 8-Prenylkaempferol-producing yeast strain was constructed to efficiently generate 8-prenylkaempferol from glucose via introducing 11 heterologous genes and modifying 12 native yeast genes. The yield of 
icaritin produced by co-culturing S. cerevisiae and E. coli was $19.70 \mathrm{mg} / \mathrm{L}$, which was higher than that of mono-culture S. cerevisiae $(7.20 \mathrm{mg} / \mathrm{L}$, as described in 4.1) [57]. By dividing the long synthetic pathway into different microbial strains, it provides a better environment for specific enzymes or the overexpression of some pathway genes, minimizes feedback inhibition, and reduces the pressure of metabolic flux.

In addition to free aglycones being synthesized by co-culture strategy, some glycosylated flavonoids have also been successfully synthesized through synthetic microbial consortia. Different from free flavonoids, the synthesis process of glycosylated flavonoids needs to introduce some glycosyltransferases to modify these compounds and provide sufficient sugar groups. For example, co-culture systems were developed in two E. coli strains to produce apigetrin (Apigenin-7-O-b-D-glucoside). An upstream strain containing 4CL from Nicotiana tabacum, CHS from P. hybrida, CHI from M. sativa, and FNS from Parsley specially synthesized apigenin from $p$-coumaric acid, and the downstream strain was responsible for the overproduction of UDP-glucose and heterologous expression of a glycosyltransferase (PaGT3) to convert apigenin into apigetrin [87]. By optimizing the initial inoculum ratio of strains, temperature, and media component, the titer of apigetrin could reach $16.60 \mathrm{mg} / \mathrm{L}$. Meanwhile, this co-culture strategy restricted the formation of undesired byproducts. Another typical example is the de novo biosynthesis of pelargonidin-3-Oglucoside by the mixed culture of four different $E$. coli strains. De novo biosynthesis of pelargonidin-3-O-glucoside is difficult to be realized because of its complicated biosynthesis process and too many enzymes. In previous studies, pelargonidin-3-o-glucoside was successfully synthesized from naringin using two different engineered $E$. coli, with yields of 5.6 and $78.9 \mathrm{mg} / \mathrm{L}$, respectively [88,89]. In these studies, pelargonidin-3-o-glucoside was not synthesized de novo. Recently, de novo biosynthesis of pelargonidin-3-O-glucoside has been successfully achieved by S. cerevisiae, but the yield was low, only $0.85 \mathrm{mg} / \mathrm{L}$ [60] In the co-culture system, 15 enzymes and transcriptional factors were overexpressed on four independent $E$. coli strains, and the pelargonidin-3-O-glucoside synthesis pathway was divided into four modules [90]. The first strain was responsible for converting glucose, xylose, and glycerol into phenylpropionic acid. The second strain utilized phenylpropionic acid and exogenously added malonate to generate flavanones, which were transformed into flavan-3-ols through the third strain. The fourth strain overexpressed anthocyanin synthase (ANS) and 3-O-glycotransferase (3GT) genes to convert flavane-3-ols to anthocyanidins, and glycosylation modification of anthocyanidins, respectively. When using the synthetic microbial consortia to synthesize pelargonidin-3-O-glucoside, the optimum yield was $9.5 \mathrm{mg} / \mathrm{L}$, which was 11.2 times that of the mono-culture. These linear, binary, ternary, and even quaternary designs are used in more complex linear biosynthetic pathways to reduce the metabolic burden of each strain and enable independent strains to optimize modules for specific needs. Other examples of using a co-culture system to synthesize flavonoids are also summarized in Table 3.

Table 3. Recent examples of modular co-culture engineering for flavonoids production.

\begin{tabular}{|c|c|c|c|c|}
\hline Product & Substrate & Co-Culture System & Titer $(\mathrm{mg} / \mathrm{L})$ & Reference \\
\hline Genistein & Tyrosine & E. coli-S. cerevisiae coculture & 6 & [82] \\
\hline Genistein & Tyrosine & E. coli-S. cerevisiae coculture & 100 & [83] \\
\hline$(+)$-Afzelechin & $p$-Coumaric acid & E. coli-E. coli coculture & 40.7 & [21] \\
\hline (+)-Afzelechin & Glucose, glycerol and xylose & Three-strain E. coli polyculture & 26.1 & [90] \\
\hline Naringenin & Glucose & E. coli-E. coli coculture & 41.5 & [84] \\
\hline Naringenin & $p$-Coumaric & S. cerevisiae-S. cerevisiae coculture & 18.5 & [86] \\
\hline Naringenin & Xylose & E. coli-S. cerevisiae coculture & 21.16 & [85] \\
\hline Pelargonidin 3-O-glucoside & Glucose, glycerol and xylose & Four-strain E. coli polyculture & 9.5 & {$[90]$} \\
\hline Apigetrin & $p$-Coumaric acid & E. coli-E. coli coculture & 16.6 & [87] \\
\hline Pyranocyanidin-3-O-glucoside-phenol & $\begin{array}{l}\text { Glucose, }(+)- \\
\text { catechin and tyrosine }\end{array}$ & E. coli-E. coli coculture & 19.5 & [91] \\
\hline $\begin{array}{l}\text { Pyranocyanidin-3-O-glucoside- } \\
\text { catechol }\end{array}$ & $\begin{array}{l}\text { Glucose and }(+)- \\
\text { catechin }\end{array}$ & E. coli-E. coli coculture & 13 & [91] \\
\hline Sakuranetin & Glucose & E. coli-E. coli coculture & 79 & [92] \\
\hline Vitexin & Luteolin and apigenin & E. coli-E. coli coculture & 5050 & [93] \\
\hline Orientin & Luteolin and apigenin & E. coli-E. coli coculture & 7090 & [93] \\
\hline Icaritin & Glucose & E. coli-S. cerevisiae coculture & 19.7 & [57] \\
\hline
\end{tabular}




\section{Conclusions}

In recent times, the continuous development of synthetic biology and basic genetic engineering has not only increased the type and number of microbial hosts used to synthesize flavonoids but also increased the titer of some flavonoids. Likewise, modular co-cultivation engineering has gradually been studied for the synthesis of some natural products as an emerging method. It has some advantages in synthesizing natural products with longer biosynthetic pathways and more complex structures. At present, some flavonoids have been synthesized by co-culture strategy. Microbial co-culture strategy provides a new direction to improve the production efficiency of flavonoids and controls production cost.

Author Contributions: Investigation, H.L. (Hanghang Lou) and L.H.; resources, T.W. and H.L. (Hongyun Lu); data curation, L.H. and H.L. (Hanghang Lou); writing-original draft preparation, H.L. (Hanghang Lou); writing-review and editing, Q.C. All authors have read and agreed to the published version of the manuscript.

Funding: This study was financially supported by Public Projects of Zhejiang Province (LGF18C200003), China.

Institutional Review Board Statement: Not applicable.

Informed Consent Statement: Not applicable.

Data Availability Statement: Data sharing not applicable. No new data were created or analyzed in this study.

Conflicts of Interest: The authors declare no conflict of interest.

\section{References}

1. Wang, Y.; Chen, S.; Yu, O. Metabolic engineering of flavonoids in plants and microorganisms. Appl. Microbiol. Biotechnol. 2011, 91, 949-956. [CrossRef]

2. Lv, Y.; Marsafari, M.; Koffas, M.; Zhou, J.; Xu, P. Optimizing Oleaginous Yeast Cell Factories for Flavonoids and Hydroxylated Flavonoids Biosynthesis. ACS Synth. Biol. 2019, 8, 2514-2523. [CrossRef]

3. Kim, B.G.; Yang, S.M.; Kim, S.Y.; Cha, M.N.; Ahn, J.H. Biosynthesis and production of glycosylated flavonoids in Escherichia coli: Current state and perspectives. Appl. Microbiol. Biotechnol. 2015, 99, 2979-2988. [CrossRef]

4. Nabavi, S.M.; Samec, D.; Tomczyk, M.; Milella, L.; Russo, D.; Habtemariam, S.; Suntar, I.; Rastrelli, L.; Daglia, M.; Xiao, J.; et al. Flavonoid biosynthetic pathways in plants: Versatile targets for metabolic engineering. Biotechnol. Adv. 2020, 38, 107316. [CrossRef]

5. Brazier-Hicks, M.; Evans, K.M.; Gershater, M.C.; Puschmann, H.; Steel, P.G.; Edwards, R. The C-glycosylation of flavonoids in cereals. J. Biol. Chem. 2009, 284, 17926-17934. [CrossRef]

6. Panche, A.N.; Diwan, A.D.; Chandra, S.R. Flavonoids: An overview. J. Nutr. Sci. 2016, 5, e47. [CrossRef] [PubMed]

7. Pandey, R.P.; Parajuli, P.; Koffas, M.A.G.; Sohng, J.K. Microbial production of natural and non-natural flavonoids: Pathway engineering, directed evolution and systems/synthetic biology. Biotechnol. Adv. 2016, 34, 634-662. [CrossRef] [PubMed]

8. Birchfield, A.S.; McIntosh, C.A. Metabolic engineering and synthetic biology of plant natural products-A minireview. Curr. Plant Biol. 2020, 24. [CrossRef]

9. Maleki, S.J.; Crespo, J.F.; Cabanillas, B. Anti-inflammatory effects of flavonoids. Food Chem. 2019, 299, 125124. [CrossRef] [PubMed]

10. Kopustinskiene, D.M.; Jakstas, V.; Savickas, A.; Bernatoniene, J. Flavonoids as Anticancer Agents. Nutrients 2020, $12,457$. [CrossRef] [PubMed]

11. Weseler, A.R.; Bast, A. Masquelier's grape seed extract: From basic flavonoid research to a well-characterized food supplement with health benefits. Nutr. J. 2017, 16, 5. [CrossRef] [PubMed]

12. Shah, F.L.A.; Ramzi, A.B.; Baharum, S.N.; Noor, N.M.; Goh, H.H.; Leow, T.C.; Oslan, S.N.; Sabri, S. Recent advancement of engineering microbial hosts for the biotechnological production of flavonoids. Mol. Biol. Rep. 2019, 46, 6647-6659. [CrossRef] [PubMed]

13. Li, J.; Tian, C.; Xia, Y.; Mutanda, I.; Wang, K.; Wang, Y. Production of plant-specific flavones baicalein and scutellarein in an engineered E. coli from available phenylalanine and tyrosine. Metab. Eng. 2019, 52, 124-133. [CrossRef]

14. Xu, P.; Marsafari, M.; Zha, J.; Koffas, M. Microbial Coculture for Flavonoid Synthesis. Trends Biotechnol. 2020, 38, 686-688. [CrossRef] [PubMed]

15. Shrestha, A.; Pandey, R.P.; Dhakal, D.; Parajuli, P.; Sohng, J.K. Biosynthesis of flavone C-glucosides in engineered Escherichia coli. Appl. Microbiol. Biotechnol. 2018, 102, 1251-1267. [CrossRef] 
16. Rodriguez, A.; Strucko, T.; Stahlhut, S.G.; Kristensen, M.; Svenssen, D.K.; Forster, J.; Nielsen, J.; Borodina, I. Metabolic engineering of yeast for fermentative production of flavonoids. Bioresour. Technol. 2017, 245, 1645-1654. [CrossRef]

17. Levisson, M.; Patinios, C.; Hein, S.; de Groot, P.A.; Daran, J.M.; Hall, R.D.; Martens, S.; Beekwilder, J. Engineering de novo anthocyanin production in Saccharomyces cerevisiae. Microb. Cell Fact. 2018, 17, 103. [CrossRef] [PubMed]

18. Hwang, E.I.; Kaneko, M.; Ohnishi, Y.; Horinouchi, S. Production of plant-specific flavanones by Escherichia coli containing an artificial gene cluster. Appl. Environ. Microbiol. 2003, 69, 2699-2706. [CrossRef] [PubMed]

19. Dunstan, M.S.; Robinson, C.J.; Jervis, A.J.; Yan, C.; Carbonell, P.; Hollywood, K.A.; Currin, A.; Swainston, N.; Feuvre, R.L.; Micklefield, J.; et al. Engineering Escherichia coli towards de novo production of gatekeeper (2S)-flavanones: Naringenin, pinocembrin, eriodictyol and homoeriodictyol. ACS Synth. Biol. 2020, 5, ysaa012. [CrossRef] [PubMed]

20. Marin, L.; Gutierrez-Del-Rio, I.; Entrialgo-Cadierno, R.; Villar, C.J.; Lombo, F. De novo biosynthesis of myricetin, kaempferol and quercetin in Streptomyces albus and Streptomyces coelicolor. PLoS ONE 2018, 13, e0207278. [CrossRef]

21. Jones, J.A.; Vernacchio, V.R.; Sinkoe, A.L.; Collins, S.M.; Ibrahim, M.H.A.; Lachance, D.M.; Hahn, J.; Koffas, M.A.G. Experimental and computational optimization of an Escherichia coli co-culture for the efficient production of flavonoids. Metab. Eng. 2016, 35, 55-63. [CrossRef]

22. Zhou, S.; Lyu, Y.; Li, H.; Koffas, M.A.G.; Zhou, J. Fine-tuning the (2S)-naringenin synthetic pathway using an iterative highthroughput balancing strategy. Biotechnol. Bioeng. 2019, 116, 1392-1404. [CrossRef]

23. Pandey, R.P.; Malla, S.; Simkhada, D.; Kim, B.G.; Sohng, J.K. Production of 3-O-xylosyl quercetin in Escherichia coli. Appl. Microbiol. Biotechnol. 2013, 97, 1889-1901. [CrossRef] [PubMed]

24. Khan, M.K.; Zill, E.H.; Dangles, O. A comprehensive review on flavanones, the major citrus polyphenols. J. Food Compos. Anal. 2014, 33, 85-104. [CrossRef]

25. Peterson, J.J.; Beecher, G.R.; Bhagwat, S.A.; Dwyer, J.T.; Gebhardt, S.E.; Haytowitz, D.B.; Holden, J.M. Flavanones in grapefruit, lemons, and limes: A compilation and review of the data from the analytical literature. J. Food Compos. Anal. 2006, 19, S74-S80. [CrossRef]

26. Pei, J.; Dong, P.; Wu, T.; Zhao, L.; Cao, F.; Tang, F. Characterization flavanone $3 \beta$-hydroxylase expressed from Populus euphratica in Escherichia coli and its application in dihydroflavonol production. Appl. Biochem. Microbiol. 2017, 53, 318-324. [CrossRef]

27. Kang, J.H.; McRoberts, J.; Shi, F.; Moreno, J.E.; Jones, A.D.; Howe, G.A. The flavonoid biosynthetic enzyme chalcone isomerase modulates terpenoid production in glandular trichomes of tomato. Plant Physiol. 2014, 164, 1161-1174. [CrossRef] [PubMed]

28. Zhang, T.; Yuan, W.; Li, M.; Miao, M.; Mu, W. Purification and characterization of an intracellular alpha-l-rhamnosidase from a newly isolated strain, Alternaria alternata SK37.001. Food Chem. 2018, 269, 63-69. [CrossRef]

29. Lee, J.W.; Kim, N.H.; Kim, J.Y.; Park, J.H.; Shin, S.Y.; Kwon, Y.S.; Lee, H.J.; Kim, S.S.; Chun, W. Aromadendrin Inhibits Lipopolysaccharide-Induced Nuclear Translocation of NF-kappaB and Phosphorylation of JNK in RAW 264.7 Macrophage Cells. Biomol. Ther. 2013, 21, 216-221. [CrossRef]

30. Ma, C.; Yang, L.; Wang, W.; Yang, F.; Zhao, C.; Zu, Y. Extraction of dihydroquercetin from Larix gmelinii with ultrasound-assisted and microwave-assisted alternant digestion. Int. J. Mol. Sci. 2012, 13, 8789-8804. [CrossRef]

31. Kiehlmann, E.; Li, E.P.M. ISOMERIZATION OF DIHYDROQUERCETIN. J. Nat. Prod. 1995, 58, 450-455. [CrossRef]

32. Monfoulet, L.E.; Ruskovska, T.; Ajdzanovic, V.; Havlik, J.; Vauzour, D.; Bayram, B.; Krga, I.; Fabiola, C.J.K.; Kistanova, E.; Abadjieva, D.; et al. Molecular Determinants of the Cardiometabolic Improvements of Dietary Flavanols Identified by an Integrative Analysis of Nutrigenomic Data From a Systematic Review of Animal Studies. Mol. Nutr. Food Res. 2021, e2100227. [CrossRef] [PubMed]

33. Ruskovska, T.; Massaro, M.; Carluccio, M.A.; Arola-Arnal, A.; Muguerza, B.; Vanden Berghe, W.; Declerck, K.; Bravo, F.I.; Calabriso, N.; Combet, E.; et al. Systematic bioinformatic analysis of nutrigenomic data of flavanols in cell models of cardiometabolic disease. Food Funct. 2020, 11, 5040-5064. [CrossRef]

34. Lee, K.W.; Kim, Y.J.; Lee, H.J.; Lee, C.Y. Cocoa has more phenolic phytochemicals and a higher antioxidant capacity than teas and red wine. J. Agric. Food Chem. 2003, 51, 7292-7295. [CrossRef]

35. Karabay, A.; Saija, J.D.; Field, D.T.; Akyurek, E.G. The acute effects of cocoa flavanols on temporal and spatial attention. Psychopharmacology 2018, 235, 1497-1511. [CrossRef] [PubMed]

36. Martin, M.A.; Ramos, S. Impact of cocoa flavanols on human health. Food Chem. Toxicol. 2021, 151, 112121. [CrossRef] [PubMed]

37. Khoo, H.E.; Azlan, A.; Tang, S.T.; Lim, S.M. Anthocyanidins and anthocyanins: Colored pigments as food, pharmaceutical ingredients, and the potential health benefits. Food Nutr. Res. 2017, 61, 1361779. [CrossRef] [PubMed]

38. Iwashina, T. Flavonoid Properties of five Families newly Incorporated into the Order Caryophyllales (Review). Bull. Natl. Mus. Nat. Sci. Ser. B Bot. 2013, 39, 25-51.

39. Castañeda-Ovando, A.; Pacheco-Hernández, M.d.L.; Páez-Hernández, M.E.; Rodríguez, J.A.; Galán-Vidal, C.A. Chemical studies of anthocyanins: A review. Food Chem. 2009, 113, 859-871. [CrossRef]

40. Orlikova, B.; Tasdemir, D.; Golais, F.; Dicato, M.; Diederich, M. Dietary chalcones with chemopreventive and chemotherapeutic potential. Genes Nutr. 2011, 6, 125-147. [CrossRef]

41. Michalkova, R.; Mirossay, L.; Gazdova, M.; Kello, M.; Mojzis, J. Molecular Mechanisms of Antiproliferative Effects of Natural Chalcones. Cancers 2021, 13, 2730. [CrossRef] 
42. Alves Borges Leal, A.L.; Teixeira da Silva, P.; Nunes da Rocha, M.; Marinho, E.M.; Marinho, E.S.; Marinho, M.M.; Bandeira, P.N.; Sampaio Nogueira, C.E.; Barreto, H.M.; Rodrigues Teixeira, A.M.; et al. Potentiating activity of Norfloxacin by synthetic chalcones against NorA overproducing Staphylococcus aureus. Microb. Pathog. 2021, 155, 104894. [CrossRef] [PubMed]

43. Zhang, F.X.; Yuan, Y.L.; Cui, S.S.; Li, M.; Tan, X.; Qiu, Z.C.; Li, R.M. Dissection of the potential pharmacological function of neohesperidin dihydrochalcone-A food additive-by in vivo substances profiling and network pharmacology. Food Funct. 2021, 12, 4325-4336. [CrossRef]

44. Boucherle, B.; Peuchmaur, M.; Boumendjel, A.; Haudecoeur, R. Occurrences, biosynthesis and properties of aurones as high-end evolutionary products. Phytochemistry 2017, 142, 92-111. [CrossRef] [PubMed]

45. Popova, A.V.; Bondarenko, S.P.; Frasinyuk, M.S. Aurones: Synthesis and Properties. Chem. Heterocycl. Compd. 2019, 55, 285-299. [CrossRef]

46. Sui, G.; Li, T.; Zhang, B.; Wang, R.; Hao, H.; Zhou, W. Recent advances on synthesis and biological activities of aurones. Bioorg. Med. Chem. 2021, 29, 115895. [CrossRef] [PubMed]

47. Blicharski, T.; Oniszczuk, A. Extraction Methods for the Isolation of Isoflavonoids from Plant Material. Open Chem. 2017, 15, 34-45. [CrossRef]

48. Wang, J.F.; Liu, S.S.; Song, Z.Q.; Xu, T.C.; Liu, C.S.; Hou, Y.G.; Huang, R.; Wu, S.H. Naturally Occurring Flavonoids and Isoflavonoids and Their Microbial Transformation: A Review. Molecules 2020, 25, 5112. [CrossRef]

49. Kang, H.R.; Lee, D.; Benndorf, R.; Jung, W.H.; Beemelmanns, C.; Kang, K.S.; Kim, K.H. Termisoflavones A-C, Isoflavonoid Glycosides from Termite-Associated Streptomyces sp. RB1. J. Nat. Prod. 2016, 79, 3072-3078. [CrossRef]

50. McCue, P.; Shetty, K. Health benefits of soy isoflavonoids and strategies for enhancement: A review. Crit. Rev. Food Sci. Nutr. 2004, 44, 361-367. [CrossRef] [PubMed]

51. Saldanha, G.B.; Oliveira, G.L.S.; da Silva, J.C.C.L.; Oliveira, M.C.P.; da Silva, A.P.S.C.L.; de Lima David, J.P. Neoflavonoids as Prospective Compounds Against Parasitic Neglected Tropical Infections and Human Immunodeficiency Virus. Curr. Bioact. Compd. 2017, 13. [CrossRef]

52. Garazd, M.M.; Garazd, Y.L.; Khilya, V.P. Neoflavones. 1. Natural distribution and spectral and biological properties. Chem. Nat. Compd. 2003, 39, 54-121. [CrossRef]

53. Menezes, J.; Diederich, M.F. Bioactivity of natural biflavonoids in metabolism-related disease and cancer therapies. Pharmacol. Res. 2021, 167, 105525. [CrossRef]

54. Menezes, J.; Campos, V.R. Natural biflavonoids as potential therapeutic agents against microbial diseases. Sci. Total Environ. 2021, 769, 145168. [CrossRef]

55. Gontijo, V.S.; Dos Santos, M.H.; Viegas, C., Jr. Biological and Chemical Aspects of Natural Biflavonoids from Plants: A Brief Review. Mini Rev. Med. Chem. 2017, 17, 834-862. [CrossRef]

56. Zhang, S.; Yang, J.; Li, H.; Chiang, V.L.; Fu, Y. Cooperative Regulation of Flavonoid and Lignin Biosynthesis in Plants. Crit. Rev. Plant Sci. 2021, 40, 109-126. [CrossRef]

57. Wang, P.; Li, C.; Li, X.; Huang, W.; Wang, Y.; Wang, J.; Zhang, Y.; Yang, X.; Yan, X.; Wang, Y.; et al. Complete biosynthesis of the potential medicine icaritin by engineered Saccharomyces cerevisiae and Escherichia coli. Sci. Bull. 2021. [CrossRef]

58. Sheng, H.; Sun, X.; Yan, Y.; Yuan, Q.; Wang, J.; Shen, X. Metabolic Engineering of Microorganisms for the Production of Flavonoids. Front. Bioeng. Biotechnol. 2020, 8, 589069. [CrossRef] [PubMed]

59. Yang, D.; Park, S.Y.; Park, Y.S.; Eun, H.; Lee, S.Y. Metabolic Engineering of Escherichia coli for Natural Product Biosynthesis. Trends Biotechnol. 2020, 38, 745-765. [CrossRef] [PubMed]

60. Eichenberger, M.; Hansson, A.; Fischer, D.; Durr, L.; Naesby, M. De novo biosynthesis of anthocyanins in Saccharomyces cerevisiae. FEMS Yeast Res. 2018, 18. [CrossRef] [PubMed]

61. Zhou, S.; Hao, T.; Zhou, J. Fermentation and Metabolic Pathway Optimization to De Novo Synthesize (2S)-Naringenin in Escherichia coli. J. Microbiol. Biotechnol. 2020, 30, 1574-1582. [CrossRef]

62. Zhou, S.; Yuan, S.F.; Nair, P.H.; Alper, H.S.; Deng, Y.; Zhou, J. Development of a growth coupled and multi-layered dynamic regulation network balancing malonyl-CoA node to enhance (2S)-naringenin biosynthesis in Escherichia coli. Metab. Eng. 2021, 67, 41-52. [CrossRef]

63. Kallscheuer, N.; Vogt, M.; Stenzel, A.; Gatgens, J.; Bott, M.; Marienhagen, J. Construction of a Corynebacterium glutamicum platform strain for the production of stilbenes and (2S)-flavanones. Metab. Eng. 2016, 38, 47-55. [CrossRef]

64. Wang, T.Y.; Tsai, Y.H.; Yu, I.Z.; Chang, T.S. Improving 3'-Hydroxygenistein Production in Recombinant Pichia pastoris Using Periodic Hydrogen Peroxide-Shocking Strategy. J. Microbiol. Biotechnol. 2016, 26, 498-502. [CrossRef]

65. Solopova, A.; van Tilburg, A.Y.; Foito, A.; Allwood, J.W.; Stewart, D.; Kulakauskas, S.; Kuipers, O.P. Engineering Lactococcus lactis for the production of unusual anthocyanins using tea as substrate. Metab. Eng. 2019, 54, 160-169. [CrossRef] [PubMed]

66. Marin, L.; Gutierrez-Del-Rio, I.; Yague, P.; Manteca, A.; Villar, C.J.; Lombo, F. De Novo Biosynthesis of Apigenin, Luteolin, and Eriodictyol in the Actinomycete Streptomyces albus and Production Improvement by Feeding and Spore Conditioning. Front. Microbiol. 2017, 8, 921. [CrossRef]

67. Liu, X.; Cheng, J.; Zhang, G.; Ding, W.; Duan, L.; Yang, J.; Kui, L.; Cheng, X.; Ruan, J.; Fan, W.; et al. Engineering yeast for the production of breviscapine by genomic analysis and synthetic biology approaches. Nat. Commun. 2018, 9, 448. [CrossRef] [PubMed] 
68. Pei, J.; Sun, Q.; Gu, N.; Zhao, L.; Fang, X.; Tang, F.; Cao, F. Production of isoorientin and isovitexin from luteolin and apigenin using coupled catalysis of glycosyltransferase and sucrose synthase. Appl. Biochem. Biotechnol. 2020, 190, 601-615. [CrossRef] [PubMed]

69. Jones, J.A.; Collins, S.M.; Vernacchio, V.R.; Lachance, D.M.; Koffas, M.A. Optimization of naringenin and p-coumaric acid hydroxylation using the native E. coli hydroxylase complex, HpaBC. Biotechnol. Prog. 2016, 32, 21-25. [CrossRef] [PubMed]

70. Lv, Y.; Edwards, H.; Zhou, J.; Xu, P. Combining 26s rDNA and the Cre-loxP System for Iterative Gene Integration and Efficient Marker Curation in Yarrowia lipolytica. ACS Synth. Biol. 2019, 8, 568-576. [CrossRef]

71. Lyu, X.; Ng, K.R.; Lee, J.L.; Mark, R.; Chen, W.N. Enhancement of Naringenin Biosynthesis from Tyrosine by Metabolic Engineering of Saccharomyces cerevisiae. J. Agric. Food Chem. 2017, 65, 6638-6646. [CrossRef]

72. Gao, S.; Lyu, Y.; Zeng, W.; Du, G.; Zhou, J.; Chen, J. Efficient Biosynthesis of (2S)-Naringenin from p-Coumaric Acid in Saccharomyces cerevisiae. J. Agric. Food Chem. 2020, 68, 1015-1021. [CrossRef]

73. Lv, Y.; Xu, S.; Lyu, Y.; Zhou, S.; Du, G.; Chen, J.; Zhou, J. Engineering enzymatic cascades for the efficient biotransformation of eugenol and taxifolin to silybin and isosilybin. Green Chem. 2019, 21, 1660-1667. [CrossRef]

74. Yang, J.; Liang, J.; Shao, L.; Liu, L.; Gao, K.; Zhang, J.L.; Sun, Z.; Xu, W.; Lin, P.; Yu, R.; et al. Green production of silybin and isosilybin by merging metabolic engineering approaches and enzymatic catalysis. Metab. Eng. 2020, 59, 44-52. [CrossRef] [PubMed]

75. Cress, B.F.; Leitz, Q.D.; Kim, D.C.; Amore, T.D.; Suzuki, J.Y.; Linhardt, R.J.; Koffas, M.A. CRISPRi-mediated metabolic engineering of E. coli for O-methylated anthocyanin production. Microb. Cell Fact. 2017, 16, 10. [CrossRef]

76. Zha, J.; Zang, Y.; Mattozzi, M.; Plassmeier, J.; Gupta, M.; Wu, X.; Clarkson, S.; Koffas, M.A.G. Metabolic engineering of Corynebacterium glutamicum for anthocyanin production. Microb. Cell Fact. 2018, 17, 143. [CrossRef] [PubMed]

77. Shrestha, B.; Pandey, R.P.; Darsandhari, S.; Parajuli, P.; Sohng, J.K. Combinatorial approach for improved cyanidin 3-O-glucoside production in Escherichia coli. Microb. Cell Fact. 2019, 18, 7. [CrossRef]

78. Koirala, N.; Pandey, R.P.; Thuan, N.H.; Ghimire, G.P.; Jung, H.J.; Oh, T.J.; Sohng, J.K. Metabolic engineering of Escherichia coli for the production of isoflavonoid-4'-O-methoxides and their biological activities. Biotechnol. Appl. Biochem. 2019, 66, 484-493. [CrossRef]

79. McCarty, N.S.; Ledesma-Amaro, R. Synthetic Biology Tools to Engineer Microbial Communities for Biotechnology. Trends Biotechnol. 2019, 37, 181-197. [CrossRef]

80. Sgobba, E.; Wendisch, V.F. Synthetic microbial consortia for small molecule production. Curr. Opin. Biotechnol. 2020, 62, 72-79. [CrossRef]

81. Wang, R.; Zhao, S.; Wang, Z.; Koffas, M.A. Recent advances in modular co-culture engineering for synthesis of natural products. Curr. Opin. Biotechnol. 2020, 62, 65-71. [CrossRef] [PubMed]

82. Katsuyama, Y.; Miyahisa, I.; Funa, N.; Horinouchi, S. One-pot synthesis of genistein from tyrosine by coincubation of genetically engineered Escherichia coli and Saccharomyces cerevisiae cells. Appl. Microbiol. Biotechnol. 2007, 73, 1143-1149. [CrossRef] [PubMed]

83. Horinouchi, S. Combinatorial biosynthesis of plant medicinal polyketides by microorganisms. Curr. Opin. Chem. Biol. 2009, 13, 197-204. [CrossRef] [PubMed]

84. Ganesan, V.; Li, Z.; Wang, X.; Zhang, H. Heterologous biosynthesis of natural product naringenin by co-culture engineering. Synth. Syst. Biotechnol. 2017, 2, 236-242. [CrossRef]

85. Zhang, W.; Liu, H.; Li, X.; Liu, D.; Dong, X.T.; Li, F.F.; Wang, E.X.; Li, B.Z.; Yuan, Y.J. Production of naringenin from D-xylose with co-culture of E. coli and S. cerevisiae. Eng. Life Sci. 2017, 17, 1021-1029. [CrossRef] [PubMed]

86. Lu, Y.; Song, Y.; Zhu, J.; Xu, X.; Pang, B.; Jin, H.; Jiang, C.; Liu, Y.; Shi, J. Potential application of CHS and 4CL genes from grape endophytic fungus in production of naringenin and resveratrol and the improvement of polyphenol profiles and flavour of wine. Food Chem. 2021, 347, 128972. [CrossRef]

87. Thuan, N.H.; Chaudhary, A.K.; Van Cuong, D.; Cuong, N.X. Engineering co-culture system for production of apigetrin in Escherichia coli. J. Ind. Microbiol. Biotechnol. 2018, 45, 175-185. [CrossRef]

88. Yan, Y.J.; Chemler, J.; Huang, L.X.; Martens, S.; Koffas, M.A.G. Metabolic engineering of anthocyanin biosynthesis in Escherichia coli. Appl. Environ. Microbiol. 2005, 71, 3617-3623. [CrossRef]

89. Yan, Y.J.; Li, Z.; Koffas, M.A.G. High-yield anthocyanin biosynthesis in engineered Escherichia coli. Biotechnol. Bioeng. 2008, 100, 126-140. [CrossRef]

90. Jones, J.A.; Vernacchio, V.R.; Collins, S.M.; Shirke, A.N.; Xiu, Y.; Englaender, J.A.; Cress, B.F.; McCutcheon, C.C.; Linhardt, R.J.; Gross, R.A.; et al. Complete Biosynthesis of Anthocyanins Using E. coli Polycultures. MBio 2017, 8, 9. [CrossRef]

91. Akdemir, H.; Silva, A.; Zha, J.; Zagorevski, D.V.; Koffas, M.A.G. Production of pyranoanthocyanins using Escherichia coli co-cultures. Metab. Eng. 2019, 55, 290-298. [CrossRef] [PubMed]

92. Wang, X.; Li, Z.; Policarpio, L.; Koffas, M.A.G.; Zhang, H. De novo biosynthesis of complex natural product sakuranetin using modular co-culture engineering. Appl. Microbiol. Biotechnol. 2020, 104, 4849-4861. [CrossRef] [PubMed]

93. Qiu, C.; Wang, H.; Zhao, L.; Pei, J. Orientin and vitexin production by a one-pot enzymatic cascade of a glycosyltransferase and sucrose synthase. Bioorg. Chem. 2021, 112, 104926. [CrossRef] [PubMed] 